\title{
Diseño de un programa en Logo para calcular flujos máximos en redes
}

\author{
M.A. Murray-Lasso \\ Unidad de Enseñanza Auxiliada por Computadora \\ D epartamento de Ingeniería de Sistemas, División de Ingeniería M ecánica e Industrial \\ Facultad de Ingeniería, UNAM \\ E-mail: mamurray@ servidor.unam.mx
}

(recibido: agosto de 2004; aceptado: diciembre de 2004)

\begin{abstract}
Resumen
Se muestran los pormenores del diseño de un programa Logo para calcular flujos máximos entre pares de puntos sobre redes orientadas, cuyas ramas tienen un límite supe rior al flujo que puede circular por ellas. Para el diseño del programa se utilizan Listas, que es la única estructura de datos soportada por Logo, pero que es muy adecuada para representar y manipular redes que no están muy densamente conectadas, que es el caso más frecuente en redes de flujo. Dado que Logo no soporta arreglos, y éstos resultan convenientes en el programa, se muestra la manera de implementarlos utilizando la flexibilidad de Logo en el manejo de los nombres de las vari ables. También se proporcionan listas de programas en Logo Writer que implementan los algoritmos de etiquetado de Ford y Fulkerson. Como apoyo a la docencia se incluyen rutinas que se pueden utilizar como instrumentos que le permiten al maestro y alumno seguir las variaciones de las estructuras durante el proceso. Finalmente, se resuelven en detalle dos ejemplos ilustrativos.
\end{abstract}

Descriptores: Logo, flujos máximos, algoritmos de etiquetado, listas, arreglos.

\begin{abstract}
Thedetails of thede sign of a Logo pro gram to cal cu latemax i mum flows between pairs of points in oriented net works with branches hav ing up per lim its to flow are presented. For the de sign Lists, which are the only data structures sup ported by Logo, but which are quite ap pro pri ate to rep re sent and handle net works not densely con nected, the most com mon casein flow net works are uti lised. Since Logo does not sup port ar rays and they are con venient in theprogram, it is shown how to im plement them taking ad van tage of theflex $i$ bil ity of Logo in thehan dling of thenames of vari ables. List ings of Logo Writer programs im plement ing thela bel ling al go rithms of F ord and Fulkerson arepro vided. As teach ingaids, rou tines that can beused as in stru ments to al low teach ers and stu dents to fol low thevari a tions in the struc tures dur ing the pro cess, arealso pro vided. Two ilustrativeex am ples are solved in det ail.
\end{abstract}

Keywords: Logo, max i mum flows, la bel ling al go rithms, lists, ar rays.

\section{Introducción}

El lenguaje Logo es uno de los lenguajes educativos diseñados para la enseñanza, incluyendo la enseñanza de la programación. Se ha popularizado entre los mae stros de educación básica y media por tener entradas fáciles al lenguaje que permiten estar programando desde la primera sesión. El lenguaje generalmente funciona como un intérprete, no tiene instrucción GOTO, es altamente estructurado, favorece la creación de procedimientos que se comunican entre sí y el uso de la recursión. El lenguaje fue creado entre la empresa Bolt, Beranek y Newman y el Grupo de Inteligencia Ar ti fi cial del MIT (Instituto Tecnológico de Massachusetts)y es un derivado del LISP. Tiene suficientes diferencias 
comparado con lenguajes como BASIC, Pascal y C que orilla al programador a pensar con un estilo diferente. Su prin ci pal (y en muchas versiones, su única) estructura de datos, es la Lista, a la cual se espera se le agreguen y eliminen los elementos exclusivamente en su principio y en su final. El lenguaje Logo ha adquirido la reputación de ser un lenguaje de programación para niños; sin embargo, el poderío del lenguaje es igual al de cualquier otro lenguaje de programación; lo que pasa es que muchos libros se concentran en (y en algunos casos se limitan a) las instrucciones elementales para dibujar con la tortuga y casi no tratan las porciones "avanzadas" del lenguaje como el manejo no trivial de listas y la recursión no triv ial.

En este artículo se diseña un programa para el cálculo del flujo máximo en una red, el cual requiere las porciones avanzadas del lenguaje. En el programa diseñado se implementan lo que equivale a vectores de listas y arreglos bidimensionales (no obstante que el lenguaje no los soporta), los cuales permiten el manejo eficiente de la memoria para redes poco densamente conectadas, que son la regla, más que la excepción en las aplicaciones. El propósito principal de este trabajo es mostrar las partes avanzadas del lenguaje Logo en un contexto de aplicación a un caso específico, en vez de hacerlo con el estilo de un manual, ilustrando con ejemplos muy cortos el uso de cada instrucción y estructura de datos.

\section{Algoritmo de etiquetado de Ford y Fulkerson para el cálculo del flujo máximo de una red}

Entre los principales pioneros de la disciplina de flujos en redes están Ford y Fulkerson (1962), quienes dieron un algoritmo para calcular el flujo máximo que puede fluir entre un nodo origen y un nodo destino en una red orientada cuyas ramas tienen una capacidad máxima de flujo. Una típica red es la que se muestra en la figura 1 , la cual utilizaremos como ejemplo du rante el artículo. En la red de la figura 1 se han numerado consecutivamente los nodos; las cantidades junto a las flechas denotan la capacidad máxima de flujo en la dirección de la flecha de la rama correspondiente. El nodo 1 es el nodo origen al cual se le inyecta un flujo que sale por el nodo destino 6. El algoritmo de Ford y Fulkerson es un algoritmo de etiquetado.

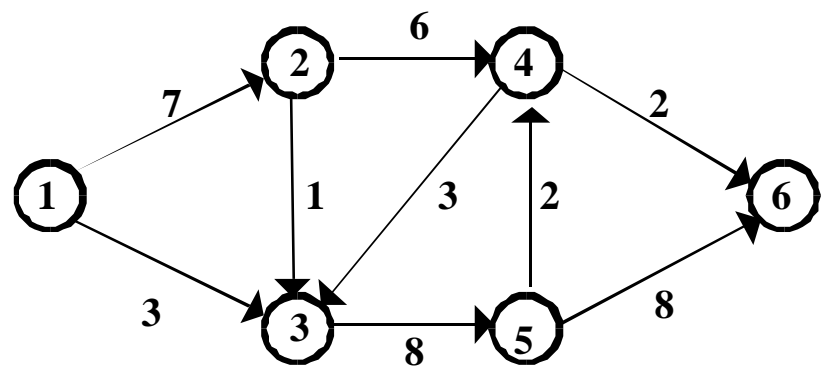

Figura 1.

Si llamamos a los nodos i, j, k, ... s, t ,... (al nodo fuente de donde parte el flujo hacia el resto de la red le llamamos sy al nodo destino al que llega el flujo del resto de la red le llamamos t); al flujo en la rama $(i, j)$ le llamamos $x_{i}$, y a la capacidad de la rama $(i, j), u_{i j}$, entonces el algoritmo es como sigue (Ford y Fulkerson, 1962):

Durante la rutina $A$, cada nodo está en uno de tres estados: no-etiquetado, etiquetado y no-rastreado, o etiquetado y rastreado. Al principio todos los nodos están en el estado no-etiquetado.

\section{Rutina A (proceso de etiquetado)}

Primero, la fuente $s$ recibe la etiqueta $\left({ }^{*}+, \varepsilon(s)=\right.$ $\infty)$. (La fuente $s$ está ahora etiquetada y no-rastreada; todos los demás nodos están no-etiquetados.) En el paso general seleccione cualquier nodo etiquetado y no-rastreado i. Supóngase que su etiqueta es $\left(z^{ \pm}, \varepsilon(i)\right)$. A todos los nodos j que están no-etiquetados y tales que el flujo $x_{i j}<u_{i j}$, asígnele la etiqueta $(i+, \varepsilon(j))$, donde

$$
\varepsilon(j)=\min \left[\varepsilon(i), u_{i j}-x_{i j}\right]
$$

(Dichas nodos j ahora están etiquetados y norastreados). A todos los nodos j que ahora están no-etiquetados, y tales que $x_{j i}>0$, asígneles la etiqueta $(i, \varepsilon(j))$, donde

$$
\varepsilon(j)=\min \left[\varepsilon(i), x_{j i}\right]
$$

(Los nodos j ahora están etiquetados y no-rastreados; i está etiquetado y rastreado).

Repítase el paso gen eral hasta que una de dos: el nodo destino $t$ esté etiquetado y no-rastreado 
o hasta que no sea posible asignar más etiquetas estando el nodo destino $t$ no-etiquetado. En el primer caso ejecute la rutina $B$, en el segundo caso termina el algoritmo.

\section{Rutina B (cambio de flujo)}

El nodo destino $t$ ha recibido la etiqueta $(j \pm, \varepsilon(t))$. Si $t$ está etiquetado $\left(j^{+}, \varepsilon(t)\right)$, reemplace $x_{j t}$ por $x_{j t}+\varepsilon(t) ;$ si t está etiquetada $\left(j^{-}, \varepsilon(t)\right)$ reemplace $x_{t j}$ por $x_{j}-\varepsilon(t)$. En cualquier caso, en seguida hay que ponerle atención al nodo j. En gen eral, si j está etiquetado $\left(i^{+}, \varepsilon(i)\right)$ reemplace $x_{i j}$ por $x_{i j}+\varepsilon(t) ; y$ si está etiquetado $\left(x^{-}, \varepsilon(j)\right)$ reemplace $x_{j i}$ por $x_{j i}-\varepsilon(t) y$ siga con el nodo i. Continúe de la misma manera hasta que alcance el nodo $s$. Elimine las etiquetas y regrese a la Rutina $A$.

El proceso de etiquetado es una búsqueda sistemática de una vereda aumentante del flujo del origen $s$ al destino $t$. Se lleva información en las etiquetas de modo que cuando se etiqueta al destino $t$ (al evento se le llama perforación), se pueden cambiar los flujos con facilidad. Si por otra parte, la Rutina A termina y el no do destino t no ha sido etiquetado (no-perforación), el flujo es máximo y los arcos que van de los nodos etiquetados a los nodos no-etiquetados son un corte mínimo, cuyo valor es igual a la suma de las capacidades de las ramas que parten de un nodo etiquetado y llegan a un nodo no-etiquetado.

\section{Descripción de la red para uso interno de la computadora}

Hay varias maneras de describir una red para uso interno en la computadora. Para redes densamente conectadas una descripción eficiente involucra lamatriz de incidencia (Ahuja et al., 1993). Dadas las características de Logo, se utilizarán las listas de predecesores y sucesores* de cada nodo. La ventaja de esta descripción es que solamente se utiliza la memoria requerida para las ramas existentes y no para todas las posibles, además de que se adapta muy bien a la estructura de datos lista con la que cuenta el lenguaje Logo. En vez de explicar la descripción en abstracto, es más fácil explicarla ejemplificando para la red de la figura 1. La red de la misma figura queda completamente especificada si se da:
1. El número de nodos.

2. El número de ramas.

3. El número del nodo origen.

4. El número del nodo destino.

5. Para cada rama de la red se da el nodo inicial, el nodo final y la capacidad máxima de flujo de la rama. Estas cantidades se pueden meter a una lista y darse en el orden descrito.

Así, para la red de la figura 1 dicha lista es:

[6916133127231246358433462542 568 ]

\section{Estructuras de datos para los sucesores y predecesores}

Para aplicar el algoritmo de Ford y Fulkerson se necesita recorrer uno a uno los sucesores y predecesores de un nodo, como paso preliminar se armarán listas de predecesores y sucesores para cada uno de los nodos. Para ello, se utilizará un vector (arreglo unidimensional) con una componente por nodo, cuyas componentes son listas de sucesores o predecesores. El lenguaje Logo no maneja arreglos; la manera como se implementarán los vectores es creando variables individuales con nombres que incluyan los índices. Así por ejemplo, llamamos sucl a la primera componente, suc2 a la segunda, ... , suc6 a la sexta, si queremos manejar un vector suc(i), $i=1, \ldots, 6$. Cada una de estas componentes será una lista con los sucesores del nodo, cuyo índice es el número al final del nombre. Esta estratagema, que sería impráctica manejarla en lenguajes como BASIC, Pascal o C, es práctica en Logo porque se puede manipular el nombre de la variable nombre en la instrucción

\section{da nombre valor}

\footnotetext{
* Son sucesoresde un nodo i los nodos j a los que se puede llegar partiendo de $i$, viajando por una sola rama en el sentido de su flecha. Son predecesores de un nodo i los nodos j a los que se puede llegar partiendo de i viajando por una sola rama en el sentido contrario a su flecha.
} 
En Logo nombre se puede construir por medio de otras instrucciones. Por ejemplo, en vez de nombre se puede escribir

\section{(palabra "suc :indice)}

y palabra se construye con las letras suc concatenadas con las cifras del valor (:) de indice. La estratagema que se de scribe es equivalente a que en los lenguajes BASIC, Pascal o $\mathrm{C}$ se tuvieran instrucciones de asignación del siguiente tipo:

"suc" + índice := expresión

aquí del lado izquierdo de la asignación se está construyendo el nombre de la variable porconcatenación. Esta instrucción no existe en los lenguajes mencionados, ya que en los tres se requiere que del lado izquierdo de una asignación aparezca literalmente el nombre legal de una vari able.

Esta posibilidad existente en Logo (y en LISP) permite el estratagema para manejar arreglos sin que el lenguaje los soporte por medio de nombres adecuadamente seleccionados de variables simples.

El siguiente procedimiento suc utiliza la estratagema indicada para construir el arreglo de sucesores de los nodos.

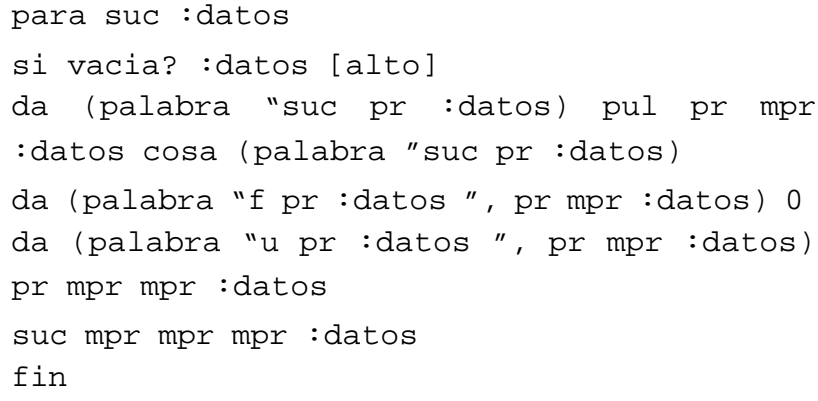

Antes de ejecutar suc (en el procedimiento datos que se muestra más abajo ) se le quitan a la lista datos (que se le pasa como parámetro al procedimiento suc), los cuatro primeros números, de modo que en datos se tiene para cada rama un trío con el nodo inicial, el nodo final y la capacidad de flujo máxima de la rama. La segunda línea de suc detiene el procedimiento (alto) cuando se vacía la lista datos, con lo cual se detiene la recursión de la última línea de suc, ya que el procedimiento se llama a sí mismo en la última línea. En la tercera línea de suc se le asigna (da) a la lista cuyo nombre (palabra) está formado por la concatenación de suc con el primer elemento (pr) de la lista datos, lo que se forma al agregar al final (pul) el segundo elemento ( $\mathrm{pr} \mathrm{mpr}$ ) de datos al resultado (cosa) del objeto cuyo nombre (palabra) es "suc concatenada con el primer elemento dedatos.

En las líneas 4 y 5 de suc se aprovecha que ya se está recorriendo la lista datos para formar e inicializar dos arreglos bidimensionales de flujos y capacidades de flujo máximo en las ramas. Se emplea la misma estratagema de ponerle a variables individuales nombres que incluyen los dos índices separados por una coma. A los flujos se le inicializa con ceros y a las capacidades se les ponen los valores tomados de la lista datos.

Todo lo que se especifica en el procedimiento suc se hace con los primeros tres elementos de la lista datos. Para hacer las operaciones con todos los demás se hace una llamada recursiva en la sexta línea cambiando el parámetro datos por la misma lista, a la cual se le eliminan sus primeros tres elementos. Esto se hace con la instrucción mpr que significa menosprimero, cuyo efecto sobre una lista es eliminar el primer elemento. Al aplicar la instrucción tres veces, el efecto es eliminar el primer trío de elementos.

En forma similar a como se procedió para formar el arreglo de sucesores, se forma un arreglo de predecesores con el procedimiento pred.

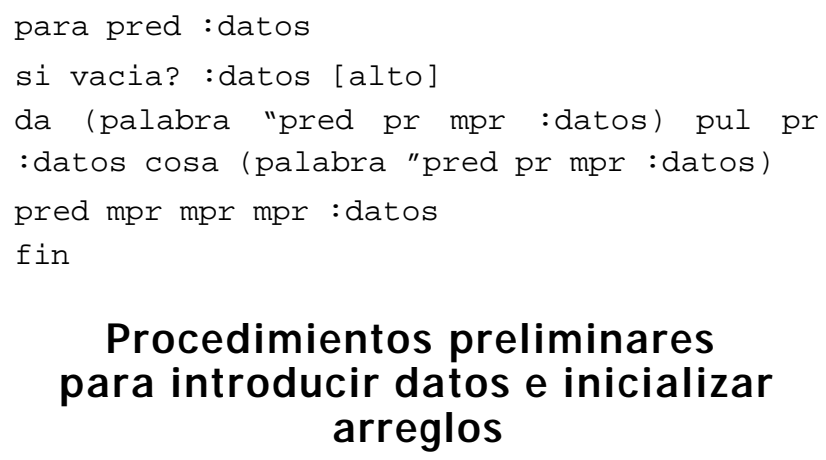

Antes de llamar a suc y pred se ejecuta el procedimiento datos, cuya función es inicializar la lista datosque de scribe la red, luego tomar los 
primeros cuatro elementos de la lista original datos, asignarle los primeros cuatro valores a las variables globales $n n, n r, n i, n f$ que representan el número de nodos, número de ramas, nodo inicial (origen) y nodo final (destino) respectivamente, y eliminar estos cuatro elementos de la lista para que en la lista datos (que es un objeto global) queden exclusivamente los tríos de las ramas.

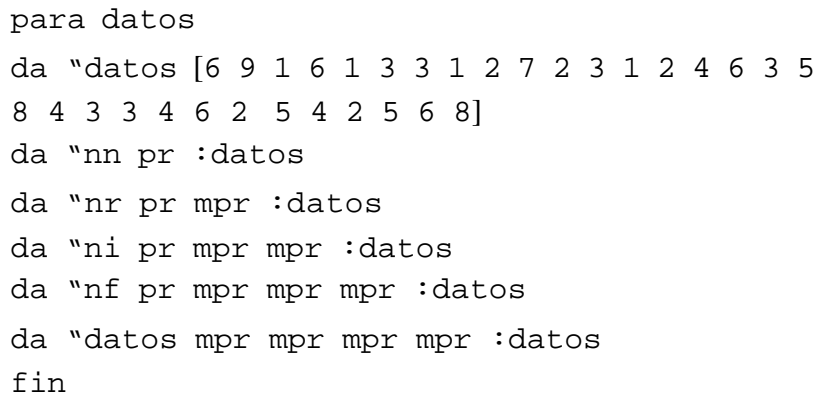

A continuación con initetiq se inicializan las etiquetas, todas con $(0,0)$, excepto el nodo origen que recibirá la etiqueta $\left({ }^{*}, 9999\right)$. El número 9999 es el número más grande que utiliza LogoWriter y lo usamos en vez de infinito $(\infty)$.

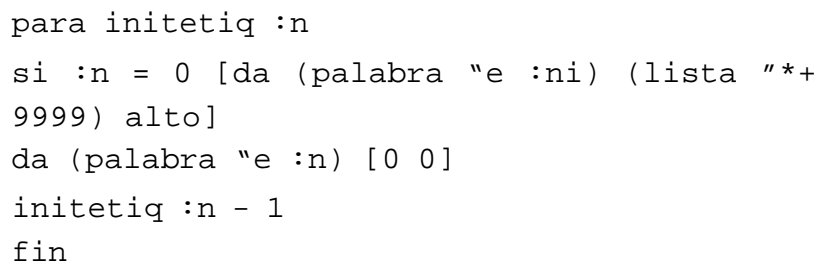

Nótese como en initetiq el cambio de índices se logra con una llamada recursiva. EI número de nodos se le proporciona al procedimiento por medio del parámetro n. Para inicializar los "arreglos" de listas de predecesores y sucesores se ejecuta el procedimiento initpredsuc, en el cual todas las listas iniciales están vacías.

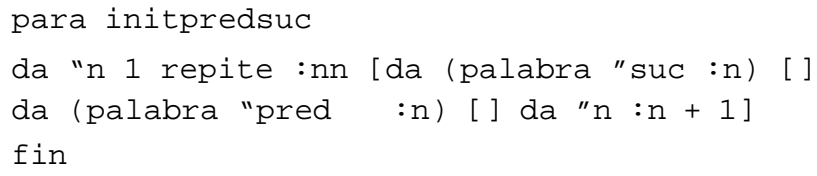

Como se indicó antes, los "arreglos" f y u para los flujos en las ramas y las capacidades de flujo máximas, se inicializan en el procedimiento suc.

\section{Procedimientos principales para calcular el flujo máximo}

Ahora se diseñarán los procedimientos principales para implementar el algoritmo de Ford y Fulkerson para el cálculo del flujo máximo. Se utilizan cuatro procedimientos : flujo, etiqsuc, etiqpred, incflujo. El primero hace la prueba para determinar si ya se ha alcanzado el flujo máximo, borra las etiquetas al final de cada perforación y reinicializa un nuevo intento para encontrar una vereda que aumente el flujo y lleva el control de la lista de los nodos etiquetados y rastreados. Los dos siguientes procedimientos (etiqsuc y etiqpred) realizan el proceso de etiquetar a todos los sucesores y predecesores del nodo al que se le está dando servicio en la lista de nodos etiquetados y no rastreados. El procedimiento incflujo hace las operaciones necesarias para incrementar el flujo en las ramas adecuadas cuando hay una perforación.

Para llevar cuenta en la computadora de cuáles nodos están etiquetados y no rastreados y cuáles están etiquetados y rastreados, se utilizan dos listas: la que almacena para cada perforación los nodos etiquetados y rastreados se llama ee y es un objeto global. La que almacena en cada perforación los nodos etiquetados y no rastreados se llama lista, se le pasa como parámetro al procedimiento flujo y está vigente en la ejecución de los tres procedimientos etiqsuc, etiqpred e incflujo. A los elementos almacenados en lista se les va dando servicio para entonces, desde ellos etiquetar sucesores y predecesores. Para que un nodo se etiquete es necesario que no esté etiquetado, es decir, que no sea miembro ni deee ni delista. Una vez que desde un nodo se etiquetan sucesores y predecesores, al nodo se le elimina de lista y se le agrega a ee. Para eliminarlo se llama recursivamente a los procedimientos etiqsuc y etiqpred con el argumento mpr lista en vez de lista. Para agregar un nodo al final de una lista se utiliza la instrucción de Logo pul elemento lista.

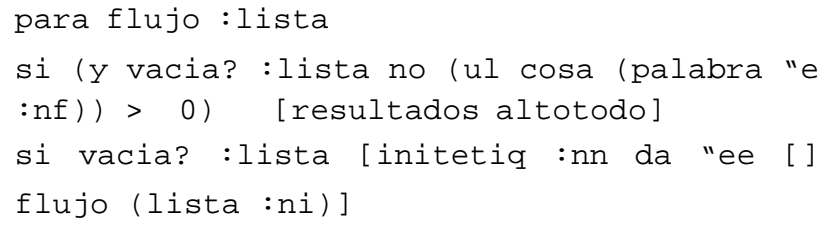




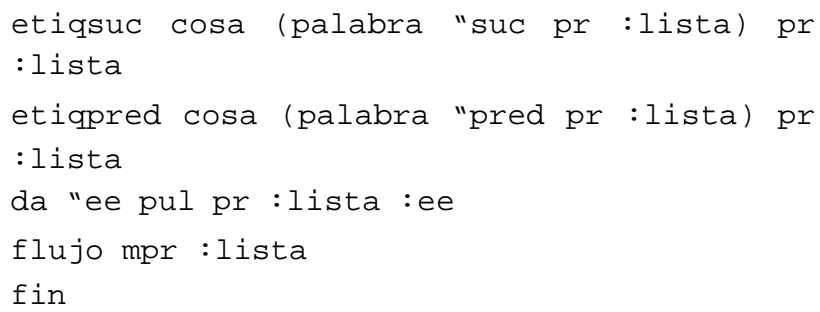

En los procedimientos etiqsuc y etiqpred se utiliza la función min, ya que el algoritmo de Ford y Fulkerson lo requiere. La función min reporta (o regresa) el mínimo de sus dos argumentos numéricos. Como LogoWriter no cuenta con esta función, ésta se ha implementado como sigue:

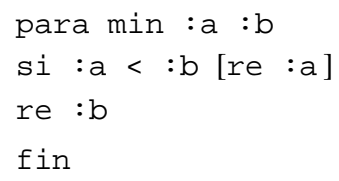

El procedimiento etiqsuc etiqueta a los sucesores del nodo al que se está dando servicio en la lista; etiqpred hace lo correspondiente para los predecesores. Detecta cuando el nodo destino recibe una etiqueta y llama al procedimiento incflujo cuando esto sucede. Controla que al regreso de esta llamada se borren y reinicialicen la lista ee, asi como las etiquetas, y se llame de nuevo al procedimiento flujo para dar servicio al primer elemento en la lista. El procedimiento incflujo se encarga de incrementar (o decrementar cuando la primera parte de la etiqueta tiene signo menos) los flujos en todas las ramas que forman una vereda del origen al destino. Para encontrar la vereda en sentido contrario, comenzando con el destino, se va fijando en las primeras partes de los nodos, cada una de las cuales apuntan al nodo etiquetador.

\section{Arranque del programa y generación de resultados}

En los procedimientos ff y calc se hacen las llamadas a los procedimientos que arrancan todo el proceso para calcular los flujos máximos. El procedimiento ff finalmente llama a los procedimientos datos, initpredsuc, suc, pred y calc. Todos, menos calc, han sido explicados anteriormente. El procedimiento calc inicializa la lista ee, llama al procedimiento que inicializa los "arreglos" de etiquetas y para arrancar los cálculos de flujo máximo, finalmente llama al procedimiento flujo para realizar los cálculos. En la llamada a flujo utiliza como parámetro lista, la misma que cuenta con un solo elemento consistente en el nodo origen de la red, ni. Cuando la 
llamada a flujo se detiene, se llama al procedimiento resultados, que imprime los flujos en las ramas que producen el flujo máximo, dando para cada rama de la red su nodo inicial, final y el valor del flujo en la rama, así como el valor del flujo máximo entre el nodo origen, destino y los nodos que especifican el corte mínimo. Para calcular los flujos máximos entre un par de nodos en una red, el usuario solamente debe, desde el editor de procedimientos de LogoWriter, colocar en el procedimiento datos la lista que de scribe la red y desde el centro de mandos teclear ff seguido de la tecla de retorno. A continuación, se muestran los procedimientos ff, calc y resultados.

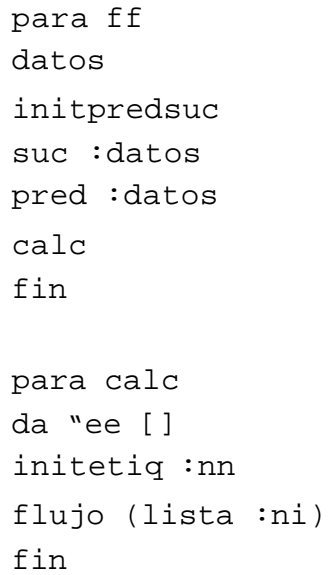

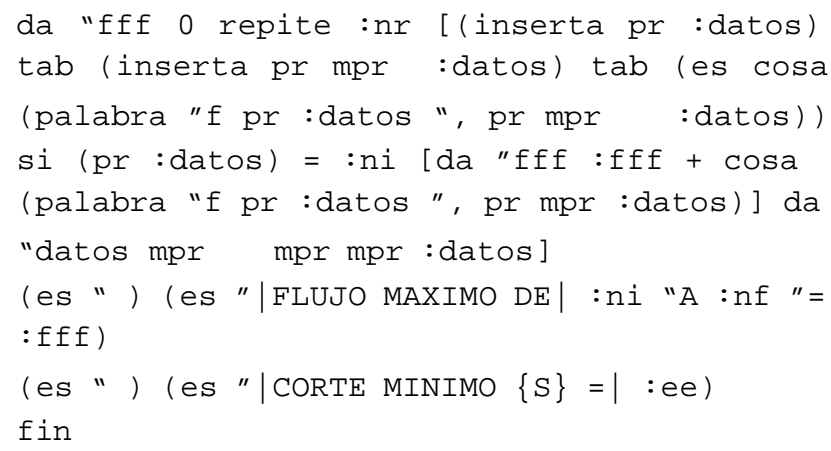

Ahora se muestra lo que producen los programas con la información del listado de datos que se anotó arriba, el cual corresponde a la red de la figura 1. El resultado se logra tecleando en el centro de mandos ff seguido de la tecla de re torno.

\section{RESULTADOS}

$\begin{array}{lll}I & F & \text { flujo } \\ 1 & & \\ 1 & 3 & 3 \\ 2 & 2 & 6 \\ 2 & 3 & 1 \\ 3 & 4 & 5 \\ 4 & 5 & 7 \\ 4 & 3 & 3 \\ 5 & 6 & 2 \\ 5 & 4 & 0 \\ & 6 & 7\end{array}$

FLUJO MAXIMO DE 1 A $6=7$ CORTE MINIMO $\{\mathrm{S}\}=124$

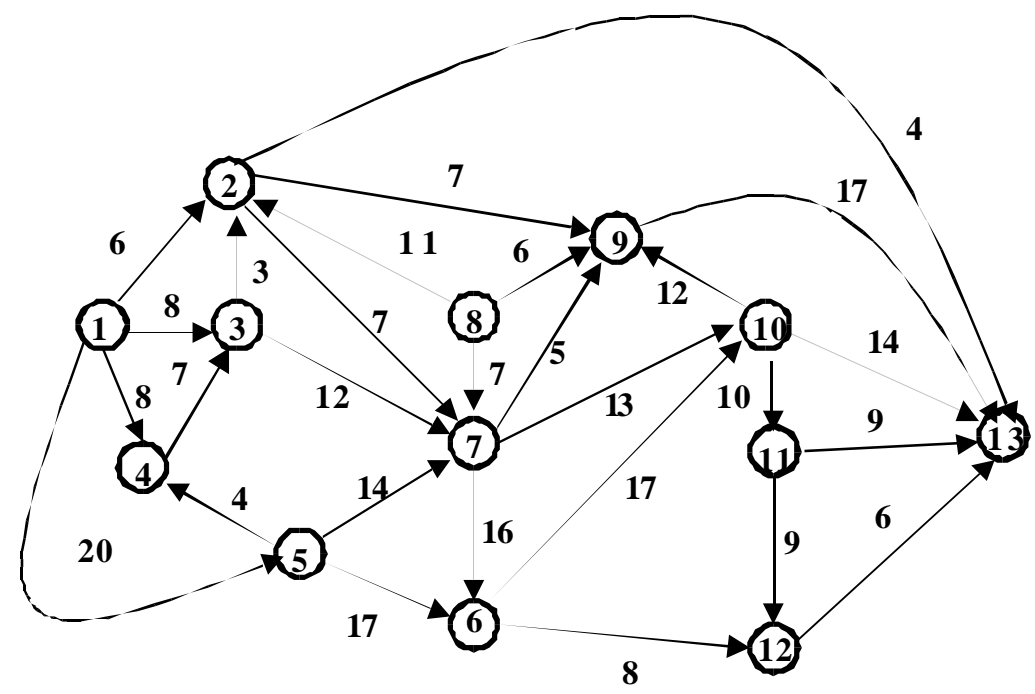

Figura 2. 


\section{Ejemplo adicional}

Para probar el programa con una red un poco mayor, se resolverá un ejemplo tomado de (Jensen y Barnes, 1980). La red se muestra en la figura 2. Junto a cada rama aparecen sus capacidades de flujo máximas. Si se toma como nodo origen el nodo 1 y como nodo destino el 13, notamos que la red tiene 13 nodos y 28 ramas. La representación de la red en forma de lista es la siguiente:

$\left[\begin{array}{lllllllllllllllllllll}13 & 28 & 1 & 13 & 1 & 2 & 6 & 1 & 3 & 8 & 1 & 4 & 8 & 1 & 5 & 20 & 2 & 7 & 7 & 2 & 9\end{array}\right.$ $\begin{array}{lllllllllllllllllllll}7 & 2 & 13 & 4 & 3 & 2 & 3 & 3 & 7 & 12 & 4 & 3 & 7 & 5 & 4 & 4 & 5 & 6 & 17 & 5 & 7\end{array}$ $\begin{array}{lllllllllllllllllll}14 & 6 & 10 & 17 & 6 & 12 & 8 & 7 & 6 & 16 & 7 & 9 & 5 & 7 & 10 & 13 & 8 & 2 & 11\end{array}$ $\begin{array}{llllllllllllllllll}8 & 7 & 7 & 8 & 9 & 6 & 9 & 13 & 17 & 10 & 9 & 12 & 10 & 11 & 10 & 10 & 13 & 14\end{array}$ $\left.\begin{array}{lllllllll}11 & 12 & 9 & 11 & 13 & 9 & 12 & 13 & 6\end{array}\right]$

Esta lista se introduce en el procedimiento datos con la instrucción da "datos lista, ya sea en vez de la que actualmente aparece o a continuación (pues en este último caso la asignación simplemente sobreescribe en lista lo dejado por la anterior instrucción). Luego se teclea ff seguido de la tecla de retorno. Los resultados que se obtienen se muestran a continuación.

$\begin{array}{lll}\text { RESULTADOS } & \\ \text { I } & \text { F } & \text { flujo } \\ & & \\ 1 & 2 & 6 \\ 1 & 3 & 8 \\ 1 & 4 & 7 \\ 1 & 5 & 20 \\ 2 & 7 & 0 \\ 2 & 9 & 5 \\ 2 & 13 & 4 \\ 3 & 2 & 3 \\ 3 & 7 & 12 \\ 4 & 3 & 7 \\ 5 & 4 & 0 \\ 5 & 6 & 17 \\ 5 & 7 & 3 \\ 6 & 10 & 14 \\ 6 & 12 & 6 \\ 7 & 6 & 3 \\ 7 & 9 & 5 \\ 7 & 10 & 7 \\ 8 & 2 & 0 \\ & & \end{array}$

$\begin{array}{lll}8 & 7 & 0 \\ 8 & 9 & 0 \\ 9 & 13 & 17 \\ 10 & 9 & 7 \\ 10 & 11 & 0 \\ 10 & 13 & 14 \\ 11 & 12 & 0 \\ 11 & 13 & 0 \\ 12 & 13 & 6\end{array}$

FLUJO MAXIMO DE 1 A $13=41$

CORTE MINIMO $\{S\}=14$

Revisando los resultados constatamos:

1) que se satisfacen los límites máximos sobre todos los flujos,

2) que se satisface la Ley de Conservación de Flujos de Kirchhoff en cada uno de los nodos habiendo un exceso de flujo que sale del nodo origen igual al exceso de flujo que llega al nodo destino, ambos con valor igual a la capacidad 0 valor del corte mínimo, el cual corresponde a las ramas que atraviesan la superficie gaussiana que encierra los nodos 1 y 4 . La teoría de flujos nos dice que basta que se satisfagan las restricciones máximas de flujo en todas las ramas, la ley de conservación de flujo en todos los nodos y que el flujo entre origen y destino sea igual al valor de algún corte con respecto a origen y destino para que dicha distribución de flujos corresponda a un flujo máximo entre origen y destino. De esta manera, se tiene la certeza de que la solución producida por el programa es correcta.

\section{Instrumentación adicional para el programa}

El programa desarrollado en este artículo tiene propósitos educativos, por ello, conviene agregarle instrumentos adicionales que sean útiles en la de teoría de flujos en redes, en general y de la enseñanza del algoritmo de Ford y Fulkerson para el cálculo de flujos máximos, en particular. Ante todo, conviene que el alumno pueda ver las estructuras de datos en lugares estratégicos del programa para comparar con lo que el alumno haría manualmente. Algunas estructuras de datos 
no cambian du rante to do el proceso, por lo que se pueden imprimir una vez. Este es el caso de las listas de predecesores y sucesores y las variables nn, nr, ni, nf con la cantidad de nodos, ramas, el nodo origen y el nodo destino, así como las capacidades máximas de flujo de las ramas y sus nodos inicial y final.

Las cantidades que varían durante el curso de la aplicación del algoritmo son: las etiquetas de los nodos, los flujos en las ramas, el flujo total entre origen y destino.

Se utilizarán los siguientes procedimientos auxiliares: $\mathbf{n}, \mathbf{p}, \mathbf{s}, \mathbf{c}, \mathbf{e}, \mathbf{f}, \mathbf{e e}, \mathbf{l i}$, los cuales imprimen respectivamente las cantidades de nodos, ramas, nodo inicial y final; las listas de predecesores, las listas de sucesores, las capacidades de flujo máximo en las ramas, las etiquetas de los nodos, los flujos en las ramas obtenidos hasta el momento y el flujo total de origen a destino obtenido hasta el momento; el contenido de la lista œ de nodos etiquetados y rastreados hasta el momento; y el contenido de la lista lista con los nodos etiquetados y no rastreados hasta el momento. son:

Los listados de los procedimientos auxiliares

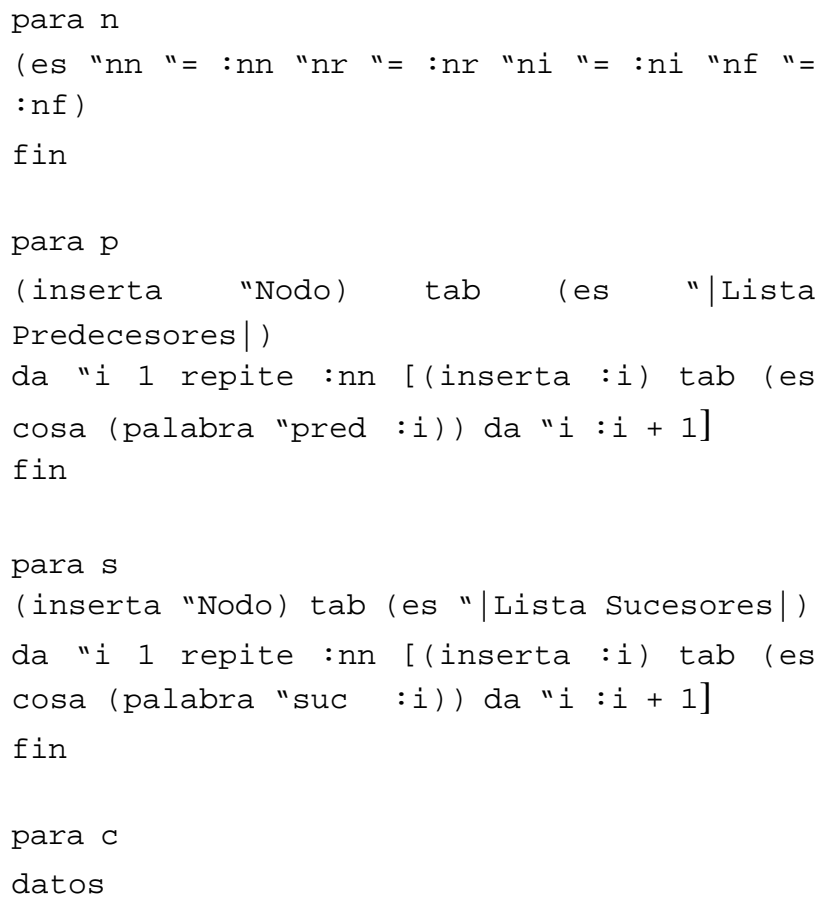

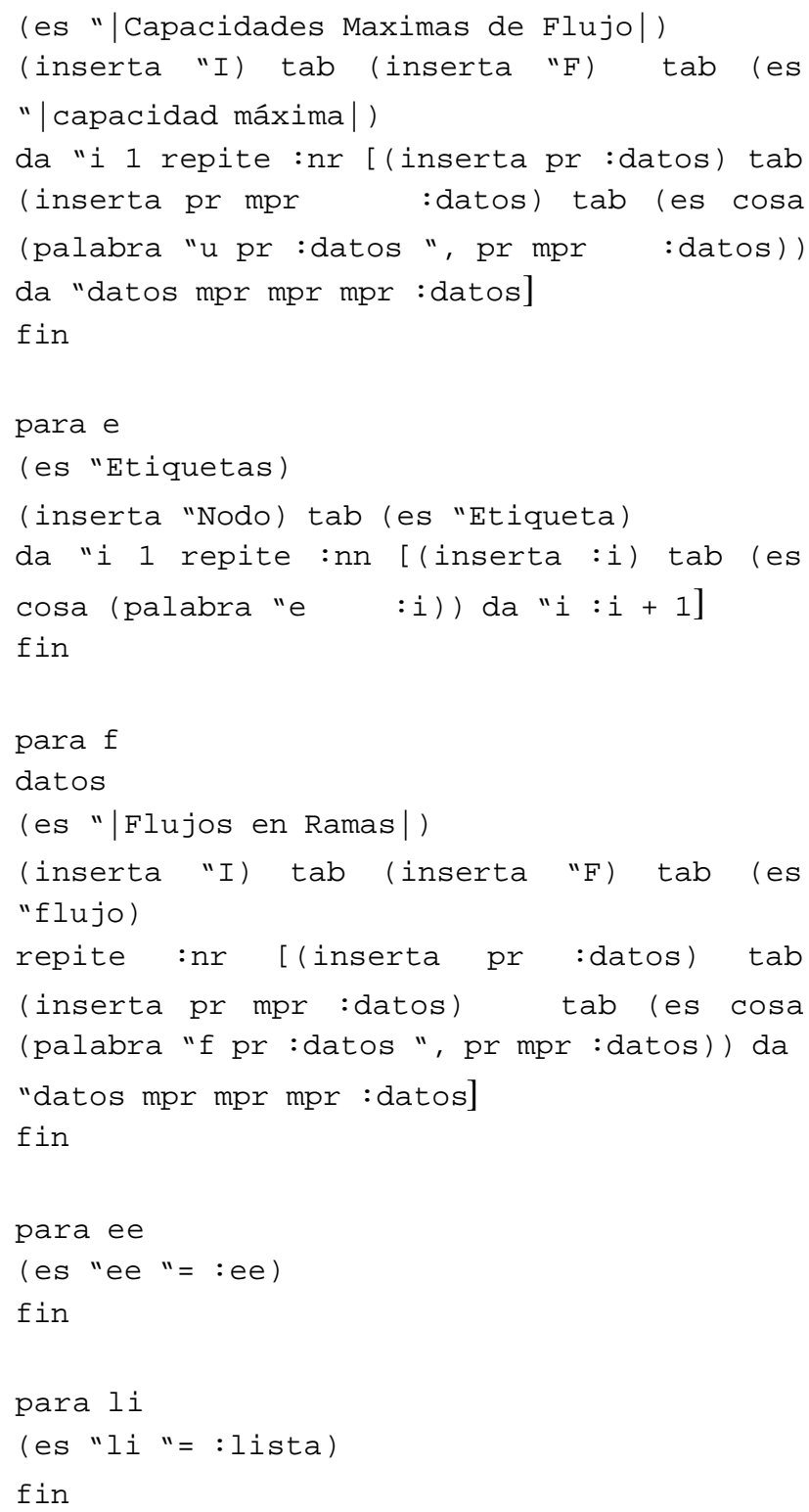

Se pueden insertar llamadas a los procedimientos en lugares estratégicos del programa para visualizar el proceso algorítmico y la manera como cambian los resultados intermedios.

A con tinuación se in dica una posibilidad:

Llamadas a $\mathbf{n}$ : En el procedimiento calc inmediatamente antes de flujo (lista :ni)

Llamadas a p: Inmediatamente después de la llamada a $\mathbf{n}$.

Llamadas a s. Inmediatamente después de la llamada a $\mathbf{p}$.

Llamadas a c: Inmediatamente después de la llamada a s. 
Llamadas a e En el procedimiento etiqsuc inmediatamente después del "[" en la línea que comienza si (ul cosa etc. En el procedimiento etiqpred inmediatamente después del "[" en la línea que comienza si (ul cosa etc.

Llamadas a f: En el procedimiento incflujo inmediatamente después del "[" en la segunda línea que comienza si $: \mathbf{n n}=$ etc.

Llamadas a ee: En el procedimiento flujo inmediatamente antes de la penúltima línea que dice flujo mpr :lista.

Llamadas a li: En el procedimiento flujo inmediatamente antes de la línea que comienza etiqsuc cosa etc.

A continuación, se muestra un segmento corto de una parte intermedia de la salida producida por el programa cuando está instrumentado con los procedimientos listados arriba y con las llamadas colocadas en los lugares indicados. Los datos corresponden al la red de la figura 2.

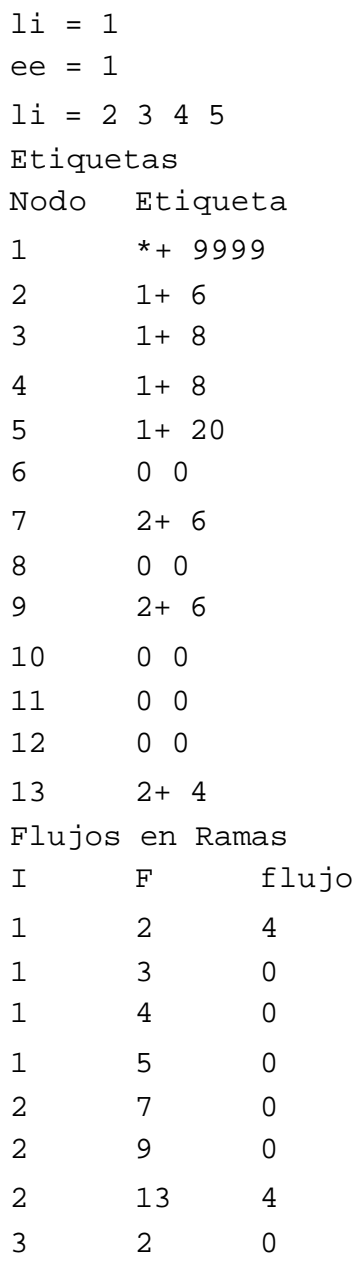

\begin{tabular}{|c|c|c|c|}
\hline & 7 & & 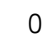 \\
\hline 4 & 3 & & 0 \\
\hline 5 & 4 & & 0 \\
\hline 5 & 6 & & 0 \\
\hline & 7 & & 0 \\
\hline 6 & 10 & & 0 \\
\hline 6 & 12 & & 0 \\
\hline 7 & 6 & & 0 \\
\hline 7 & 9 & & 0 \\
\hline 7 & 10 & & 0 \\
\hline 8 & 2 & & 0 \\
\hline 8 & 7 & & 0 \\
\hline 8 & 9 & & 0 \\
\hline 9 & 13 & & 0 \\
\hline 10 & 9 & & 0 \\
\hline 10 & 11 & & 0 \\
\hline 10 & 13 & & 0 \\
\hline 11 & 12 & & 0 \\
\hline 11 & 13 & & 0 \\
\hline 12 & 13 & & 0 \\
\hline$l i=1$ & & & \\
\hline ee $=1$ & & & \\
\hline $1 i=2$ & 3 & & \\
\hline ee $=1$ & 2 & & \\
\hline $1 i=3$ & 4 & 5 & 9 \\
\hline ee $=1$ & 2 & 3 & \\
\hline $1 i=4$ & 5 & 7 & \\
\hline$e e=1$ & 2 & 3 & \\
\hline $1 i=5$ & 7 & 9 & \\
\hline ee $=1$ & 2 & 3 & 5 \\
\hline$l i=7$ & 9 & 6 & \\
\hline ee $=1$ & 2 & 3 & 5 \\
\hline$i=9$ & 6 & 10 & \\
\hline
\end{tabular}

\section{Conclusiones}

Se ha presentado el diseño y realización de un programa en el lenguaje Logo para el cálculo de flujo máximo en una red orientada con capacidades de flujo máximo en las ramas. El programa se basa en el algoritmo de Ford y Fulkerson. La estrategia encuentra veredas aumentantes de flujo en orden de número de ramas, por lo que se puede garantizar la eficiencia cúbica del algoritmo, a diferencia del algoritmo original de Ford y Fulkerson que podría tardarse un tiempo exponencial (Ahuja et al., 1993). Se desarrolló el programa en Logo con el propósito de ilustrar el poderío de dicho lenguaje en aplicaciones en las que nor malmente se utilizan lenguajes como FORTRAN, Pas- 
cal, C o BASIC, los cuales soportan arreglos uni y multidimensionales, así como algunos de ellos (Pascal y C), listas ligadas y apuntadores. Logo no so porta arreglos; sin em bargo, se puede implantar el equivalente a los mismos por medio de distintas vari ables para cada componente, incorporando los índices en el nombre de la vari able. En el artículo se muestra la manera de hacerlo. De esta forma, se puede ahorrar memoria e incluir solamente las variables que aparecen en la red, las cuales en aplicaciones re ales son un porcentaje muy bajo de las posibles. Por otra parte, los componentes de los "arreglos" que se manejan en el artículo, frecuentemente son listas de longitud vari able durante el curso del programa, y las listas si son estructuras de datos que maneja bien el lenguaje Logo. En este artículo se proporciona un listado completo del programa en LogoWriter con una discusión detallada de su implantación y dos redes resueltas en todo detalle. Aquí no se pretende enseñar LogoWriter. El lector que requiera ayuda con las instrucciones puede consultar Logo Computer Sys tems Inc., 1990. Para hacer más útil el programa en la educación, también se proporciona un juego de instrumentos educativos (procedimientos) para que el mae stro y estudiante puedan observar el curso del algoritmo, como los cambios en las estructuras de datos para poder comparar con lo que se obtendría al aplicar manualmente el algoritmo de Ford y Fulkerson.
El autor espera que después de leer este artículo, el lector quede convencido que el lenguaje Logo no es sólo un lenguaje para niños, sino un lenguaje completo que tiene algunas entradas simples, desde las cuales lo pueden abordar los niños pero que les puede servir para crecer intelectualmente y utilizarlo el resto de su vida en aplicaciones tan complejas como aquellas en las que utiliza otros lenguajes de computadora.

\section{Referencias}

Ahuja R.K., Magnanti T.L. y Orlin J.B. (1993). $\mathrm{N}$ etwork Flows: Theory, Algo rithms, and Appli cations. Prentice-Hall, Upper Saddle River, NJ.

Ford Jr. L.R. y Fulkerson D.R. (1962). Flows in Networks. Princeton University Press, Princeton, NJ.

Jensen P.A. y Barnes J.W. (1980). N etwork Flow Programming. John Wiley $\&$ Sons, Inc., New York, pp. 165.

Logo Computer Systems, Inc. (1990). LogoWriter: Guía de Referencia. Macrobit Editores, México.

\section{Bibliografía sugerida}

Cristofides N. (1975). Graph Theory an Algo rithmic Approach. Academic Press, Inc., Londres.

\section{Semblanza del autor}

M arco A ntonio M urray-Lasso. Realizó la licenciatura en ingeniería mecánica-eléctrica en la Facultad de Ingeniería de la UNAM . EI Instituto de Tecnología de Massachussetts (MIT) le otorgó los grados de maestro en ciencias en ingeniería eléctrica y doctor en ciencias cibernéticas. En México, ha laborado como investigador en el Instituto de Ingeniería y como profesor en la Facultad de Ingeniería (UNAM) durante 44 años; en el extranjero, ha sido asesor de la NASA en diseño de circuitos por computadora para aplicaciones espaciales, investigador en los Laboratorios Bell, así como profesor de la Universidad Case Western Reserve y Newark College of Engineering, en los Estados Unidos. Fue el presidente fundador de la Academia Nacional de Ingeniería de México; vicepresidente y presidente del Consejo de Academias de Ingeniería y Ciencias Tecnológicas (organización mundial con sede en Washington que agrupa las Academias Nacionales de hgeniería) y secretario de la Academia Mexicana de Ciencias. Actualmente es jefe de la Unidad de Enseñanza Auxiliada por Computadora de la Secretaría de Estudios de Posgrado de la Facultad de Ingeniería de la UNAM, investigador nacional, consejero educativo del MIT y consultor de la UNESCO, así como Presidente Fundador del Consejo de Honor de la Academia Mexicana de Ciencias, Artes, Tecnología y Humanidades. 